\title{
Temporomandibular disorders and Burnout syndrome in students of the eighth period of dentistry course
}

\author{
Disfunção temporomandibular e sindrome de Burnout em \\ estudantes do oitavo período do curso de odontologia
}

\author{
Laís Valencise MAGRI ${ }^{1}$ 0000-0001-8050-4396 \\ Melissa de Oliveira MELCHIOR ${ }^{1}$ (DD 0000-0003-4943-1242 \\ Larissa JARINA $^{1}$ iD 0000-0001-8422-9141 \\ Flávia Fanchiotti SIMONAGGIO ${ }^{1}$ iD 0000-0001-5150-0169 \\ César BATAGLION ${ }^{1}$ iD 0000-0001-6634-8569
}

\section{ABSTRACT}

Objective: To investigate the incidence of painful temporomandibular disorders (TMD) and Burnout Syndrome (BS) in Dentistry students, based on the subjective perception of signs and symptoms, and to verify if there is a coexistence relation between these conditions. Methods: 57 students ( $8^{\text {th }}$ period of the dentistry course, 23 years mean age), 39 women and 18 men, responded to the Maslach Burnout Inventory Questionnaire - Students Survey (MBI-SS), ProTMDMulti and were evaluated for the presence of TMD diagnosis (DC/TMD). Results: The incidence of TMD was $22 \%$ and BS was $8.7 \%$. Among the students with BS, $80 \%$ had a TMD diagnosis, as well as a greater subjective perception of TMD signs and symptoms and a longer duration of pain. They also showed higher scores of signs/symptoms perception for situations involving orofacial function. Conclusion: There seems to be a clinical coexistence between the BS and painful TMD in the studied sample, Dentistry students with BS and high scores on the MBI-SS are more likely to have painful TMD.

Indexing terms: Burnout. Facial pain. Students, dental. Temporomandibular joint disorders.

\section{RESUMO}

Objetivo: Investigar a incidência de disfunção temporomandibular dolorosa (DTM) e Síndrome de Burnout (SB) em estudantes de Odontologia, com base na percepção subjetiva de sinais e sintomas, e verificar se há uma relação de coexistência entre estas duas condições. Métodos: 57 estudantes ( $8^{\circ}$ período, média de idade de 23 anos), 39 mulheres e 18 homens, responderam ao Inventário de Burnout de Maslach - Versão para Estudantes (MBI-SS), ProDTMMulti e foram avaliados quanto à presença de DTM (DC/TMD). Resultados: A incidência de DTM foi de $22 \%$ e de SB de 8,7\%. Dentre os estudantes com SB, $80 \%$ apresentavam diagnóstico de DTM, bem como maior percepção subjetiva de sinais e sintomas de DTM e maior tempo de duração da dor. Estes estudantes também apresentaram escores mais elevados quanto à percepção de sinais e sintomas em situações que envolviam as funções orofaciais. Conclusão: Parece haver uma relação clínica de coexistência entre a SB e a DTM dolorosa na amostra estudada, estudantes de Odontologia com SB e elevados escores no MBI-SS estão mais propensos a apresentarem DTM dolorosa.

Termo de indexação: Esgotamento psicológico. Estudantes de Odontologia. Dor facial.

$\boldsymbol{\nabla} \boldsymbol{\nabla} \nabla$

1 Universidade de São Paulo, Faculdade de Odontologia de Ribeirão Preto, Departamento de Odontologia Restauradora. Av. Café, s/n., 14040904, Ribeirão Preto, SP, Brasil. Correspondence to: MO MELCHIOR. E-mail: <mmelchior@forp.usp.br>.

$\mathbf{v} \boldsymbol{v} \mathbf{v}$

How to cite this article

Magri LV, Melchiori MO, Jarina L, Simonagio FF, Bataglion C. Temporomandibular disorders and Burnout syndrome in students of the eighth period of dentistry course. RGO, Rev Gaúch Odontol. 2019;67:e20190055. http://dx.doi.org/10.1590/1981-863720190005520180011 


\section{INTRODUCTION}

Temporomandibular disorder (TMD) is currently understood as a musculoskeletal pain syndrome, with overlapping comorbidities of physical signs and symptoms, as well as changes in behavior, emotional status, and social interactions [1,2]. The main predictors for the development of painful TMD are the presence of other painful conditions, non-painful orofacial symptoms (e.g., selfreport of parafunctions), frequency of somatic symptoms, poor sleep quality, and genetic and epigenetic factors [2]. Among comorbidities, those related to emotional states, such as anxiety and depression, have higher relevance in the perception of painful TMD [1-3].

TMD patients and/or other musculoskeletal pain syndromes frequently present inappetence, fatigue, signs and symptoms of anxiety and stress, sleep disturbances, labor difficulties, and loss of general quality of life $[4,5]$. Recent studies have shown that psychological factors such as catastrophizing, lack of assertiveness in requesting help, and delay in seeking treatment related to the fear of the unknown may be associated with the maintenance of TMD pain $[6,7]$.

In this sense, burnout syndrome (BS) is characterized by a psychological reaction due to emotional tension, which leads to a reduction of motivation, interest, and abilities regarding professional activities/studies, causing serious negative consequences in the individual's social, professional, and family life $[8,9]$. The prevalence of this syndrome in health care professionals is high, varying between 15 and 47\% [10]. Some studies have shown that in dentistry students, this prevalence varies between 7 and 20\% [11-14].

Clinical evaluation of the emotional aspects in the identification and management of TMD is of great importance because they directly affect the chronification of pain $[6,7]$. Therefore, it is necessary to understand the association between BS and TMD, which few researchers have explored in the scientific literature. In this perspective, the objective of this study was to investigate the incidence of temporomandibular disorders and Burnout Syndrome in undergraduate students in Dentistry, based on the subjective perception of signs and symptoms, to verify if there is a coexistence relationship between these two conditions in this sample.

\section{METHODS}

This manuscript shows the partial results of a prospective cohort study, with a duration of five years, carried out with graduate students in dentistry, followed up on annually. Some results of this cohort have already been published [15]. The total sample consisted of 57 students of the 8th period of the dentistry course of the School of Dentistry of Ribeirão Preto, University of São Paulo (FORP/ USP), aged between 17 to 28 years, after approval from the ethics committee (CAAE: 24347113.7.0000.5419). The inclusion criterion, therefore, was to be studying the 8th period of dentistry, and the exclusion criteria was the presence or history of neurological or psychiatric disorders. The students responded to the Maslach Burnout Inventory - Students Survey (MBI-SS) questionnaire [16] and the Protocol for Multi-Professional Centers for the Determination of the Signs and Symptoms of Temporomandibular Disorders (ProTMDMulti) $[17,18]$. In addition, the presence of TMD was verified based on the Diagnostic Criteria for Temporomandibular Disorders (DC/ TMD) evaluation protocol [19].

The MBI-SS questionnaire consists of three dimensions: emotional exhaustion (EE), depersonalization (DP), and personal accomplishment (PA). Each of these dimensions is composed of questions that represent the emotional state, the interest/mood, and the perception of the capacity to competently perform activities related to vocational training, respectively. The answers obtained are scored based on a Likert scale, often used in self-perception questionnaires, which ranges from 0 to 6 , where zero $=$ never, $1=$ almost never, 2 = sometimes, 3 = regularly, $4=$ often, $5=$ almost always, and $6=$ always. Because this is a classification based on the self-perception of this particular population sample, cuts were scored in each dimension for the judgments of high/low scores. These cuts were established from the 66th percentile (P66) for EE and DP, and 33 (P33) for PA, as proposed by Maslach and Jackson [8], totaling 19 subjects in each dimension. Thus, students who presented mean values above P66 for EE and DP and below P33 for PA concomitantly were considered BS patients.

The ProTMDMulti is a questionnaire that investigates the self-perception of 10 signs and symptoms frequently reported in the literature related to TMD in 4 daily situations: waking, chewing, speaking, and resting (i.e., without chewing or speaking). It presents a numerical scale from 0 (absence) to 10 (worst possible severity) for each sign/symptom. The scores can vary from 0 to 100 in 
each situation, and it has a maximum score of 400 when adding the scores of the 4 situations investigated. The higher the score, the greater the severity perceived. The analyses carried out considered the sum of the 4 situations to obtain the maximum score of each subject $[17,18]$.

Both questionnaires were applied simultaneously. The students' approach was carried out in the classroom, where they were oriented about the application of the questionnaires and the objectives of the research. The time taken to answer them was on average 15 minutes. Responsible researchers remained in the classroom throughout the entire process to address any doubts that might arise.

One-way ANOVA (Tukey post-test) was used to compare the group of students with BS with the subgroups of isolated dimensions with values of above P66 for EE and DP and below P33 for PA regarding the scores of the ProTMDMulti in the moments "wake up," "chewing," "speaking," "resting,", total score and difficulty to chew food. For the correlation analysis of TMD diagnosis with self-perception of its symptomatology (ProTMDMulti) as well as BS, the Pearson's correlation test was used, with a significance level of $5 \%$.

\section{RESULTS}

The mean age of the students was $23 \pm 2.5$ years, of whom 39 were women and 18 were men. After analyzing the percentiles of each dimension of the MBI-SS, 5 students (8.7\%) obtained average scores above the cutoff values for the three dimensions, being considered indicative of BS. Regarding the other dimensions, subgroups of students were analyzed who obtained mean scores above the percentiles established for each dimension alone, and each subgroup was composed of 19 students according to P66 for EE and DP and P33 for PA. In these subgroups, the majority were women, such as in the EE subgroup, which included 17 women and only two men (table 1).

Table 1. Sample composition ( $\mathrm{n}$, age, sex), percentage of TMD diagnoses and subtypes according to the Diagnostic Criteria for Temporomandibular Disorders (DC/TMD), pain duration (months), ProDTMMulti - total score. Correlation between the TMD diagnoses (DC/TMD) and the ProTMDMulti - total score. Correlation between the TMD diagnoses (DC/TMD) and the MBI-SS (Burnout Syndrome) (Pearson Correlation).

\begin{tabular}{|c|c|c|c|c|c|}
\hline & Total & Burnout & $\begin{array}{c}\text { EE > } 4.6 \\
\text { (Percentil 66) }\end{array}$ & $\begin{array}{c}\text { DP }>2 \\
\text { (Percentil 66) }\end{array}$ & $\begin{array}{c}\text { PA }<3.67 \\
\text { (Percentil 33) }\end{array}$ \\
\hline$n$ & 57 & $5(8.7 \%)$ & 19 & 19 & 19 \\
\hline Sex & $\begin{array}{c}39 \mathrm{~F} \\
18 \mathrm{M}\end{array}$ & $\begin{array}{l}4 \mathrm{~F} \\
1 \mathrm{M}\end{array}$ & $\begin{array}{l}17 \mathrm{~F} \\
2 \mathrm{M}\end{array}$ & $\begin{array}{l}12 \mathrm{~F} \\
7 \mathrm{M}\end{array}$ & $\begin{array}{l}12 \mathrm{~F} \\
7 \mathrm{M}\end{array}$ \\
\hline $\begin{array}{l}\text { Subtypes of TMD diagnoses } \\
\text { (DC/TMD) }\end{array}$ & & $\begin{array}{c}-\operatorname{MP}(2) \\
-\operatorname{LM}(1) \\
-\operatorname{MP} \text { e AR (1) }\end{array}$ & $\begin{array}{c}\text { - HATMD (3) } \\
\text { - LM (1) } \\
\text { - MP (1) } \\
\text { - MP e DDWR (1) } \\
\text { - MP, AR e DDWR (1) } \\
\text { - HATMD e DDWR (1) }\end{array}$ & $\begin{array}{c}-\operatorname{LM}(1) \\
-\operatorname{AR}(1) \\
- \text { DJD (1) } \\
- \text { MP e AR (1) } \\
\text { - MP, AR e DDWR (1) }\end{array}$ & $\begin{array}{c}-\operatorname{MP}(1) \\
-\operatorname{LM}(1) \\
-\operatorname{DJD}(1) \\
-\mathrm{MP} \text { e DDWR (1) } \\
\text { - MP e AR (1) } \\
\text { - MP, AR e DDWR (1) }\end{array}$ \\
\hline ProTMDMulti - total score & & $67.4(46)$ & $43.7(37)$ & $28.3(34)$ & $34.2(38)$ \\
\hline $\begin{array}{l}\text { Correlation between the TMD diagnoses } \\
\text { (DC/TMD) and the ProTMDMulti - total } \\
\text { score }\end{array}$ & & $\begin{aligned} p & =0.03^{*} \\
r & =0.71\end{aligned}$ & $\begin{aligned} p & =0.04^{*} \\
r & =0.69\end{aligned}$ & $\begin{array}{c}p=0.007^{*} \\
r=0.59\end{array}$ & $\begin{array}{l}p=0.008^{*} \\
r=0.75\end{array}$ \\
\hline $\begin{array}{l}\text { Correlation between the TMD } \\
\text { diagnoses (DC/TMD) and the MBI-SS } \\
\text { (Burnout Syndrome) }\end{array}$ & & $\begin{array}{c}p=0.03^{*} \\
r=0.9\end{array}$ & $\begin{array}{c}p=0.0004^{*} \\
r=0.73\end{array}$ & $\begin{array}{c}p<0.0001^{*} \\
r=0.86\end{array}$ & $\begin{array}{l}p=0.83 \\
r=0.04\end{array}$ \\
\hline
\end{tabular}

Note: LM - local myalgia, MP - myofascial pain, AR - arthralgia, HATMD - headache attributed to TMD, DDwR - disc displacement with reduction, DJD - degenerative joint disease. "Statistical significance, $p<0.05, r=$ Pearson correlation coefficient. 
The diagnosis of TMD according to the DC/TMD was established for the groups described previously. Among the students with BS, $80 \%$ had TMD; in the other subgroups with isolated dimensions, this percentage was lower: $42 \%$ for $\mathrm{EE}, 26 \%$ for $\mathrm{DP}$, and $31 \%$ for $\mathrm{PA}$. The painful conditions were the most commonly found (local myalgia, myofascial pain, arthralgia, and headache attributed to TMD), followed by the disc displacements with reduction. The duration of pain (months) was higher for the BS group (72 months) in relation to the other subgroups of isolated dimensions (34 for EE, 22 for DP, and 18 for PA). The same pattern was observed in the total score of the ProTMDMulti, that is, students with BS had higher scores than the other subgroups (table 1).

A positive correlation was found between the presence of TMD diagnosis and the total score of the ProTMDMulti for all analyzed groups $(p<0.05)$, showing that students who were diagnosed with TMD according to the DC/TMD also had a higher subjective perception of signs and symptoms of pain, joint noises, and limitation of mandibular mobility during orofacial functions. In addition, a correlation was found between the MBI-SS scores and the presence of a TMD diagnosis. For the BS group, the association found was with the total MBI-SS score (inverted PA score, $p=0.03)$, and for the EE subgroup ( $p=0.004)$ and DP $(p<0.001)$, the association was with the mean score of each dimension (table 1).

The students with the presence of BS showed higher scores in relation to the other subgroups for the following ProTMDMulti evaluations: "chewing" $(p=0.04)$, "speaking" ( $p=0.03)$, difficulty in chewing food (0.03), and total score (0.01). For the "waking" and "resting" moments, there were no significant differences between groups (table 2).

The scatter plot of Figure 1 shows the correlation between the total score of the MBI-SS and ProTMDMulti

Table 2. Intergroup comparisons (Burnout group and subgroups of isolated dimensions) of the perception of TMD signs and symptoms (ProTMDMulti) at the following moments: "waking", "chewing", "speaking" and "resting", in addition to the total ProTMDMulti score and difficulty to chew.

\begin{tabular}{|c|c|c|c|c|c|c|}
\hline & Total score & Waking & Chewing & Speaking & Resting & Difficulty to chew \\
\hline Burnout & $67.4(46)$ & $18.6(13)$ & $21(12)$ & 15 (13) & $12.8(11)$ & $31.4(16)$ \\
\hline$E E>4.6$ & 43.7 (37) & $15.3(12)$ & $12.1(10)$ & $8(10)$ & $8.3(9)$ & $22.4(12)$ \\
\hline$D P>2$ & $28.3(34)$ & $11.3(10)$ & $7.6(10)$ & $4.1(9)$ & $5.3(8)$ & $21.6(12)$ \\
\hline$P A<3.67$ & $34.2(38)$ & $11.7(12)$ & $8.6(10)$ & $6.3(10)$ & $7.6(9)$ & $21(11)$ \\
\hline$p^{*}$ & $0.01^{* *}$ & 0.09 & $0.04^{* *}$ & $0.03^{* *}$ & 0.1 & $0.03^{* *}$ \\
\hline
\end{tabular}

Note: "ANOVA (One-Way), ${ }^{* *}$ Statistically significant difference of the Burnout group in relation to the other isolated dimensions.

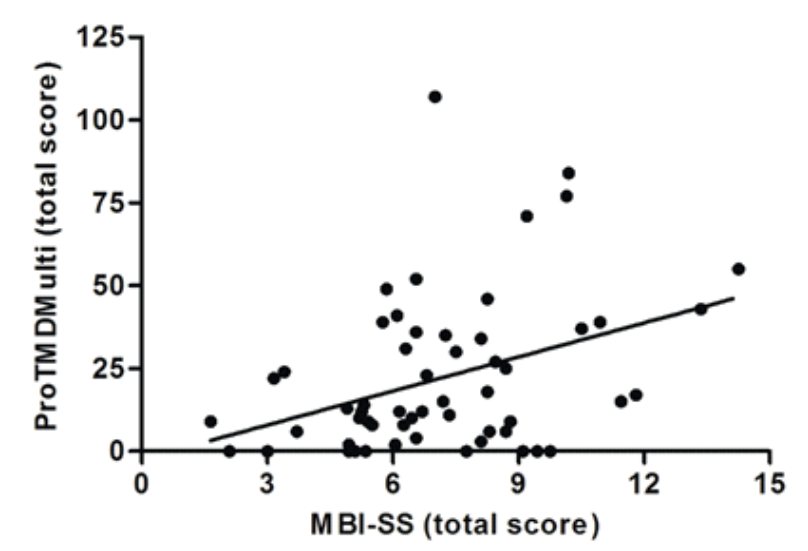

Figure 1. Scatter plot that represents the coexistence relation between the subjective perception of TMD signs and symptoms (ProTMDMulti, total score) and Burnout Syndrome (MBI-SS, total score). Correlation of Pearson, $p=0.03, r=0.89$.
( $p=0.03, r=0.89)$, revealing that students who perceive themselves with higher scores of BS dimensions also have higher subjective perception of TMD signs and symptoms at several times of the day, as well as higher difficulty in chewing certain types of foods.

\section{DISCUSSION}

The results of the present study revealed that $8.7 \%$ of dentistry students showed an indication of BS presence, with higher three-dimensional percentiles when compared to the cutoff values established for the sample. A study conducted with Brazilian dentistry students found a 17\% prevalence of BS [11]. Other studies with a similar sample profile observed a prevalence of BS between 7 and 20\% 
[12-14]. The fact of being enrolled in an undergraduate course in the health area is considered a risk factor for BS development [10]. After the academic training period, the levels of stress, anxiety, and BS in newly formed dentists remain high and can be perpetuated throughout their professional life [20].

Of the students who showed percentiles above the cutoff values, the majority were women. This result may be related to the composition of the sample, with a higher predominance of women studying dentistry, but it may indicate a trend of higher prevalence of BS in the female gender, which also shows a higher incidence and prevalence of painful TMD $[1,2]$. Some studies have observed that men have less personal accomplishment, whereas women have greater emotional exhaustion [11,21]. In this sense, a previous study demonstrated a correlation between emotional exhaustion and the perception of TMD signs and symptoms in women dentists [15]. However, Alemany Martinez et al. found a higher prevalence of BS in men, which may be related to the fact that women seek help and family support more quickly compared to men [21]. Campos et al. [11] stated that BS does not have a significant relationship with gender because other factors are more relevant in the manifestation of this condition: performance in the course, medication use due to studies, and thoughts about leaving the course.

The diagnosis of TMD was obtained for $80 \%$ of the students with BS, whereas in the other subgroups of isolated dimensions, this percentage was lower but still important very significant: $42 \%$ for $E$ E, $26 \%$ for DP, and $31 \%$ for PA. These results indicate a clinical association between BS and its dimensions with TMD, which was confirmed by the correlation test (Pearson), through which a positive correlation was observed between the total MBISS score and the presence of TMD diagnosis (DC/TMD). In a previous study of this same group, an association was observed between the subjective perception of TMD signs and symptoms (ProTMDMulti) and emotional exhaustion for women, as well as an increase in the frequency of indications of BS and the risk of developing it over the years [15]. Despite the low personal accomplishment found for men, this dimension did not correlate with the total ProTMDMulti score, suggesting a differentiation between men and women in the manifestation of the most affected dimensions for each gender [15]. Perhaps BS is a risk factor for TMD that is higher for women than for men because of the students who showed indications of BS, $80 \%$ were female and $20 \%$ were male. However, for such confirmation a specific investigation about BS as a risk factor for TMD is necessary, as a case-control study delineation for example.

In addition, the duration of pain was higher for the BS group (72 months) when compared to the other subgroups of isolated dimensions ( 34 for EE, 22 for DP, and 18 for PA). A recent review showed that the most prevalent and significant factors associated with BS in dentistry are the following: young age, student status, high number of study/work hours, involvement with clinical practice, and certain types of personality [22]. These factors are related to the emergence of emotional impairment, such as stress, anxiety, and depression, which are important comorbidities associated with painful TMD [3,23]. Emotional factors such as catastrophization and anxiety may influence the expression of pain processes, amplifying the nociceptive sensitization [3].

The correlation found between the total score of the ProTMDMulti and the presence or absence of a diagnosis of TMD according to the DC/TMD indicates a higher subjective perception of TMD signs and symptoms in students who did present the condition. The correlation between the MBI-SS scores with the ProTMDMulti scores and the presence of TMD diagnosis emphasizes the comprehensiveness of this condition in the emotional aspects present in the sample studied.

The students with the presence of BS showed higher scores than the other subgroups for the following ProTMDMulti evaluations: "chewing" ( $p=0.04)$, "speaking" (0.03), difficulty chewing food (0.03), and total score (0.01). For the "waking" and "resting" moments, there were no significant differences between the groups. Considering that $80 \%$ of these students showed confirmation of the diagnosis of TMD (DC/TMD), the findings of higher perception of TMD signs and symptoms under functional conditions are justified because it is characterized by musculoskeletal changes that manifest pain, which worsens in the mandibular function [24].

The limitations of this study were the following: The MBI-SS points to an indication of the presence of BS but does not establish a diagnosis, which must be done by a mental health professional; this study was carried out in a population with particular characteristics, so the results cannot be directly extrapolated to other populations, such as students from non-health courses; the DC/TMD diagnoses TMD subtypes based on information from the 
last 30 days, so it is not possible to infer that this association is always the same over time. Although the study statistic has demonstrated the presence of an association between these two variables, it is not possible to affirm that there is a direct association between them, since they are clinical conditions with multiple intrinsic variables involved. The confirmation of how they are correlated requires investigation by specific design, such as a case-control study, to investigate other variables of higher importance in the clinical manifestation of both conditions: stress and anxiety, student's performance profile in the course, phenotypes chronic pain, quality of life, sociocultural level, coping strategies of adverse conditions, among many others.

\section{CONCLUSION}

There is a clinical coexistence between BS, especially the dimensions of emotional exhaustion and depersonalization, with the presence of TMD diagnosis in dentistry students. The severity of TMD signs and symptoms according to subjective perception is higher in students with BS, followed by the subgroup of the emotional exhaustion dimension. Since painful TMD is modulated by biopsychosocial factors, Dentistry students with the concomitant occurrence of both can be a complicating factor for the management of TMD signs and symptoms and deserves attention. Clinical relevance: BS leads to an emotional impairment, decrease in motivation, interest, and ability to perform professional or student activities. Painful TMD is modulated by biopsychosocial factors, therefore the coexistence between BS and TMD seems possible and deserves attention in the identification and treatment of these two conditions. The concomitant occurrence of both can be a complicating factor for the management of TMD signs and symptoms, especially pain.

\section{Collaborators}

LV MAGRI, participated in the execution of this work, contributing to the elaboration of the project, data collection, writing of the manuscript and statistical analysis. MO MELCHIOR, participated in the execution of this work, contributing to the elaboration of the project, data collection and writing of the manuscript. L JARINA, participated in the execution of this work, contributing to the literature review, data collection and tabulation. FF SIMONAGGIO, participated in the execution of this work, contributing to the literature review, data collection and tabulation. C BATAGLION, participated in the execution of this work, contributing to the elaboration of the project, infrastructure, discussion of the findings and revision of the manuscript.

\section{REFERENCES}

1. Ohrbach R, Dworkin SF. The Evolution of TMD Diagnosis: Past, Present, Future. J Dent Res. 2016;95(10):1093-101. http://dx.doi.org/10.1177/0022034516653922

2. Slade GD, Ohrbach R, Greenspan JD. Painful Temporomandibular Disorder: Decade of Discovery from OPPERA Studies. J Dent Res. 2016;95(10):1084-92. http:// dx.doi.org/10.1177/0022034516653743

3. Dıraçoğlu D, Yıldırım NK, Saral İ, Özkan M, Karan A, Özkan $\mathrm{S}$, et al. Temporomandibular dysfunction and risk factors for anxiety and depression. J Back Musculoskelet Rehabil. 2016;29(3):487-91. http://dx.doi.org/10.3233/BMR-150644

4. Resende CM, Alves AC, Coelho LT, Alchieri JC, Roncalli AG, Barbosa GA. Quality of life and general health in patients with temporomandibular disorders. Braz Oral Res. 2013;27(2):116-21. http://doi.org/10.1590/S1806-83242013005000006.

5. Slade GD, Diatchenko L, Bhalang K, Sigurdsson A, Fillingim $R B$, Belfer I, et al. Influence of psychological factors on risk of temporomandibular disorders. J Dent Res. 2007;86(11):11205. http://dx.doi.org/10.1177 / 154405910708601119

6. Conti PC, Pinto-Fiamengui LM, Cunha CO, Conti AC. Orofacial pain and temporomandibular disorders: the impact on oral health and quality of life. Braz Oral Res. 2012;26 Suppl 1:120-3. http://dx.doi.org/10.1590/S1806-83242012000700018

7. Rollman A, Gorter RC, Visscher CM, Naeije MM. Why seek treatment for temporomandibular disorder pain complaints? A study based on semi-structured interviews. J Orofac Pain. 2013;27(3):227-34. http://dx.doi.org/10.11607/jop.1081

8. Maslach C, Jackson SE. The Measurement of Experienced Burnout. J Occup Behaviou 1981; 2(2): 99-113. https://doi. org/10.1002/job.4030020205

9. Maslach C, Jackson SE, Leiter MP. Maslach Burnout Inventory manual. 3rd ed: Consulting Psychologists Press; 1996.

10. Caballero MM, Bermejo FF, Nieto G R, Caballero MF. Prevalence and factors associated with burnout in a health area. Aten Primaria. 2001;27(5):313-7. http://dx.doi.org/10.1016/s0212-6 567(01)79373-0

11. Campos JA, Jordani PC, Zucoloto ML, Bonafé FS, Maroco J. Burnout syndrome among dental students. Rev Bras Epidemiol. 2012;15(1):155-65. http://dx.doi.org/10.1590/S14 15-790X2012000100014

12. Galán F, Ríos-Santos JV, Polo J, Rios-Carrasco B, Bullón P. Burnout, depression and suicidal ideation in dental students. Med Oral Patol Oral Cir Bucal. 2014;19(3):206-11. http:// dx.doi.org/10.4317 / medoral. 19281

13. Mafla AC, Villa-Torres L, Polychronopoulou A, Polanco $H$, Moreno-Juvinao $V$, Parra-Galvis D, et al. Burnout prevalence and correlates amongst Colombian dental students: the STRESSCODE study. Eur J Dent Educ. 2015;19(4):242-50. http://dx.doi.org/10.1111/eje.12128 
14. Atalayin C, Balkis M, Tezel H, Onal B, Kayrak G. The prevalence and consequences of burnout on a group of preclinical dental students. Eur J Dent. 2015;9(3):356-63. http://dx.doi. org/10.4103/1305-7456.163227

15. Magri LV, Melchior MO, Jarina L, Simonaggio F, Bataglion C. Relationship between temporomandibular disorder symptoms signs and Burnout syndrome among dentistry students. Rev Dor. 2016;17(3):171-77. http://dx.doi.org/10.5935/1806-00 13.20160065

16. Campos JA, Maroco J. Maslach Burnout Inventory - Student Survey: Portugal-Brazil cross-cultural adaptation. Rev Saude Publica. 2012;46(5):816-24. http://dx.doi.org/10.1590/S003 4-89102012000500008

17. De Felício CM, Mazzetto MO, de Silva MA, Bataglion C, Hotta TH. A preliminary protocol for multi-professional centers for the determination of signs and symptoms of temporomandibular disorders. Cranio. 2006;24(4):258-64. http://dx.doi.org/10.1179 / crn.2006.041

18. De Felicio CM, Melchior MO, Da Silva MAMR. Clinical validity of the protocol for multi-professional centers for the determination of signs and symptoms of temporomandibular disorders. Part II. Cranio. 2009;27(1):62-7. http://dx.doi. org/10.1179 / crn.2009.010

19. Schiffman E, Ohrbach R, Truelove $E$, et al. International RDC/TMD Consortium Network, International association for Dental Research; Orofacial Pain Special Interest Group, International Association for the Study of Pain. Diagnostic Criteria for Temporomandibular Disorders (DC/TMD) for
Clinical and Research Applications: recommendations of the International RDC/TMD Consortium Network* and Orofacial Pain Special Interest Groupt. J Oral Facial Pain Headache. 2014;28(1):6-27. http://dx.doi.org/10.11607/jop.1151

20. Kulkarni S, Dagli N, Duraiswamy P, Desai H, Vyas H, Baroudi K. Stress and professional burnout among newly graduated dentists. J Int Soc Prev Community Dent. 2016;6(6):535-541. http://dx.doi.org/10.4103/2231-0762.195509

21. Alemany MA, Berini AL, Gay EC. The Burnout Syndrome and associated personality disturbances. The study in three graduate programs in Dentistry at the University of Barcelona. Med Oral Patol Oral Cir Bucal. 2008;13(7):444-50.

22. Singh P, Aulak DS, Mangat SS, Aulak MS. Systematic review: fator contributing to burnout in dentistry. Occup Med (Lond). 2016;66(1):27-31. http://dx.doi.org/10.1093/occmed/ kqv119

23. Reissmann DR, John MT, Seedorf $H$, Doering S, Schierz $O$. Temporomandibular disorder pain is related to the general disposition to be anxious. J Oral Facial Pain Headache. 2014;28(4):322-30. http://dx.doi.org/10.11607/ofph.1277

24. Greene CS, Klasser GD, Epstein JB. Revision of the American Association of Dental Research's Science Information Statement about Temporomandibular Disorders. J Can Dent Assoc. 2010;76:115.

Received on: 26/11/2018

Final version resubmitted on: 7/2/2019

Approved on: 12/3/2019 